\title{
Substratos com compostos de adubos verdes e biofertilizante via foliar na formação de mudas de maracujazeiro-amarelo ${ }^{1}$
}

\section{Substrates with green manure compost and leaf application of biofertilizer on seedlings of yellow passion fruit plants}

\author{
Cristiane Muniz Barbosa Barros ${ }^{2 *}$; Marcelo Marques Lopes Müller ${ }^{3}$; \\ Renato Vasconcelos Botelho ${ }^{3}$; Leandro Michalovicz²; Marcelo Vicensi ${ }^{4}$; \\ Ronaldo do Nascimento ${ }^{4}$
}

\begin{abstract}
Resumo
Substratos e adubações são fundamentais para a produção de mudas, que bem nutridas produzem precocemente e resistem melhor a estresses. Estercos são muito utilizados em substratos não industrializados, com bons resultados para as mudas, mas seu custo tem se elevado. Outros resíduos podem ser utilizados para nutrir as plantas, tanto via substrato quanto via adubação foliar. O objetivo do trabalho foi avaliar substratos elaborados com compostos orgânicos de adubos verdes e o uso de adubação foliar com biofertilizante na formação de mudas de maracujazeiro-amarelo. O experimento foi conduzido em estufa entre dezembro de 2009 e fevereiro de 2010. Com delineamento de blocos casualizados e parcelas subdivididas, estudou-se adubação foliar com biofertilizante supermagro nas parcelas e os seguintes substratos nas subparcelas: solo; solo + esterco bovino; solo + composto de esterco com palha de aveia preta; solo + composto de esterco com palha de azevém; solo + composto de esterco com palha de nabo forrageiro; solo + composto de esterco com palha de ervilhaca. Foram realizadas três adubações foliares: aos 10, 25 e 40 dias após a emergência (DAE). Aos 50 DAE, as plantas foram coletadas para avaliar o crescimento e acúmulo de biomassa e nutrientes: $\mathrm{N}, \mathrm{P}, \mathrm{K}, \mathrm{Ca}$, $\mathrm{Mg}, \mathrm{Cu}, \mathrm{Mn}$ e $\mathrm{Zn}$. Os dados foram submetidos à análise de variância e teste de Tukey. O substrato solo + esterco bovino promoveu maior diâmetro de caule, altura de planta, área foliar, extensão e volume radiculares e acúmulo de nutrientes. Dentre os substratos com compostos de adubos verdes, aqueles com compostos de aveia preta e nabo forrageiro destacaram-se dos demais. $\mathrm{O}$ uso de supermagro teve resultado diverso sobre a formação das mudas, sendo benéfico quando combinado ao substrato com composto de aveia preta, e prejudicial em combinação com os substratos solo + esterco bovino e solo mais composto de nabo forrageiro. $\mathrm{O}$ acúmulo de nutrientes pelas mudas deu-se na seguinte ordem: $\mathrm{K}>\mathrm{Ca}>\mathrm{N}>\mathrm{Mg}>\mathrm{P}>\mathrm{Zn}>\mathrm{Cu}=\mathrm{Mn}$.
\end{abstract}

Palavras-chave: Passiflora edulis Sims f. Flavicarpa Degener, compostagem, supermagro

\footnotetext{
Abstract

Substrates and fertilization are fundamental for seedling production, which well nourished can produce earlier and are more resistant to stresses. Animal manures are often used in non-industrialized substrates

${ }^{1}$ Parte da Dissertação de Mestrado do primeiro autor, Universidade Estadual do Centro-Oeste, UNICENTRO, Guarapuava, PR.

${ }^{2}$ Mestres em Agronomia, Área de Produção Vegetal, UNICENTRO, Guarapuava, PR. E-mail: cris_muniz_bio@yahoo.com.br; leandroguarapuava@hotmail.com

${ }^{3}$ Profs. Drs. do Dept ${ }^{0}$ de Agronomia, UNICENTRO, Guarapuava, PR. E-mail: mmuller@unicentro.br; rbotelho@unicentro.br

${ }^{4}$ Discentes de Mestrado em Agronomia, Área de Produção Vegetal, UNICENTRO, Guarapuava, PR. E-mail: marcelo_vicensi@ hotmail.com; ronaldonasc@yahoo.com.br
}

* Autor para correspondência 
with good results, but their costs are increasing. Other residues may be used for plant nutrition, in substrates or in leaf fertilization. The aim of this work was to evaluate substrates prepared with green manure composts and the leaf application of biofertilizer on the formation of yellow passion fruit seedlings. A greenhouse experiment was conducted between December 2009 and February 2010, with a split-plot random block design. Plots received or not leaf application of supermagro biofertilizer. Subplots consisted of different substrates: soil; soil + cattle manure; soil + cattle manure composted with black oats straw; soil + cattle manure composted with ryegrass straw; soil + cattle manure composted with turnip straw; and soil + cattle manure composted with vetch straw. There were three dates of leaf fertilization: 10, 25 and 40 days after emergence (DAE). At 50 DAE plants were collected for evaluation of growth and accumulation of biomass and nutrients: $\mathrm{N}, \mathrm{P}, \mathrm{K}, \mathrm{Ca}, \mathrm{Mg}, \mathrm{Cu}, \mathrm{Mn}$ and $\mathrm{Zn}$. Data were submitted to analysis of variance and means compared by Tukey test. The substrate soil + cattle manure promoted higher stem diameter, plant height, leaf area, root length and volume and nutrient accumulation. Among substrates with green manure composts, those prepared with black oats and turnip straw outranked the others. The use of leaf biofertilizer showed diverse results on seedling formation, being beneficial when combined to substrates with black oats composted straw, and prejudicial when combined to soil + cattle manure and soil + turnip composted straw substrates. The accumulation of nutrients by the seedlings occurred in the following order: $\mathrm{K}>\mathrm{Ca}>\mathrm{N}>\mathrm{Mg}>\mathrm{P}>\mathrm{Zn}>\mathrm{Cu}=\mathrm{Mn}$.

Key words: Passiflora edulis Sims $f$. Flavicarpa Degener, composting, supermagro

\section{Introdução}

Aproximadamente 200 espécies de maracujá têm origem no Brasil, sendo economicamente mais importante o maracujá-amarelo (Passiflora edulis Sims f. Flavicarpa Degener) (MELETTI, 2000). Nas últimas décadas, a produção da fruta ganhou importância no país (FERREIRA, 2005), culminando na liderança do ranking mundial de produção (AGRIANUAL, 2010). Com 718 mil toneladas produzidas em 2009, o maracujá representa $1,5 \%$ da produção brasileira de frutas (IBGE, 2011).

Além de condições climáticas favoráveis à cultura (PIRES et al., 2009), este aumento de produção se deu em função do maior consumo nacional e internacional de frutas, e foi alcançado com incorporação de novas áreas de pomares e aumento da produtividade nas áreas já cultivadas (SILVA, 2009). Considerando, que os pomares de maracujazeiro são renovados a cada duas safras (MELETTI, 2011), toda esta situação tem gerado uma grande demanda por mudas, que de início podem definir a produção do pomar (SUSSEL, 2010).

Tanto a produtividade quanto a qualidade dos frutos estão diretamente ligadas aos tratos culturais, desde a formação das mudas, sendo o substrato e a adubação determinantes para um bom desempenho das mesmas (ARAÚJO et al., 2010; MENDONÇA et al., 2007). Um bom substrato fornece condições favoráveis ao desenvolvimento das plantas, por meio de características físico-químicas que permitam germinação e desenvolvimento radicular adequados. Um substrato ideal deve apresentar, ainda, facilidade de aquisição e transporte, ausência de patógenos, riqueza de nutrientes, $\mathrm{pH}$ adequado, boa textura e estrutura, garantindo à planta todos os nutrientes necessários (COSTA et al., 2009; DIAS; PEREIRA; SOUSA, 2007).

Com o maracujazeiro, estudaram-se substratos comerciais isolados e em mistura, sendo variável a composição em termos de fontes minerais e orgânicas e de materiais inertes (Ex. areia), embora fontes orgânicas e minerais de liberação lenta juntas registrem melhores resultados, sendo limitado o uso de inertes (PEIXOTO; PÁDUA, 1989). De acordo com Costa et al. (2008), no caso do maracujazeiro é recomendável adicionar matéria orgânica (MO) ao substrato para obter mudas de qualidade pois, além de fornecer nutrientes, ela atua como melhorador físico do solo do substrato para o crescimento radicular, aumentando o armazenamento de água e a porosidade e proporcionando equilíbrio ao sistema água-solo-planta-atmosfera (PIRES et al., 2009). Silveira et al. (2003) citam que a MO beneficia as 
mudas de maracujazeiro fornecendo ao substrato boa aeração e, também, nitrogênio, cuja carência resulta em crescimento quase nulo.

Mantendo-se a qualidade das mudas, a substituição de substratos industriais por resíduos orgânicos das propriedades pode reduzir custos de produção e gerar um novo nicho de atuação, pois as preocupações ambientais e de qualidade de vida têm favorecido o crescimento da agricultura orgânica (AO) (NEGRETTI et al., 2010), cujas mudas de pomares orgânicos, conforme a instrução normativa 64, de 18/12/2008 (MAPA, 2011), devem provir deste sistema produtivo. Assim, substratos e adubações de base orgânica estão em demanda crescente de informação, comprovada pela franca expansão da área de AO no País, terceira maior no mundo em 2007, com 32 milhões de hectares e 12 milhões de produtores (WILLER, 2011).

Esterco bovino e cama de aviário são os fertilizantes orgânicos mais utilizados, mas seu custo tem se elevado nos últimos anos, sobretudo em locais em que não há grande geração-oferta. Assim, outros resíduos, como os de adubos verdes (AV), podem servir de alternativa ou complemento, pela possibilidade de produção local. Porém, como é difícil sincronizar a liberação de nutrientes dos AV com as demandas das culturas, deve-se utilizar a compostagem para transformá-los em fertilizantes orgânicos, diminuindo-se o problema (SILVA, 2009). Dentre as espécies de AV, destacam-se as leguminosas, pela fixação biológica de $\mathrm{N}$, bem como nabo forrageiro, azevém e aveia preta, pelo bom potencial reciclador (POTT; MÜLLER; BERTELLI, 2007).

Com o desempenho da AO, os biofertilizantes vêm sendo cada vez mais utilizados, e embora haja disponibilidade de produtos comerciais, muitos biofertilizantes podem ser fabricados pelo próprio produtor, gerando economia de insumos externos e melhorias no saneamento ambiental da propriedade (DELEITO et al., 2000; MEINERZ et al., 2009). Resultante de decomposição de MO por fermentação em meio líquido, o supermagro é um biofertilizante rico em micronutrientes que, além dos efeitos nutricionais positivos, pode estimular funções vitais das plantas (PAULUS; MÜLLER; BARCELLOS, 2001). Tanaka et al. (2003) observaram maior número de tomates em plantas tratadas com micronutrientes, não havendo diferença entre adubo foliar microfol ${ }^{\circledR}$ (micronutrientes) e supermagro. Souza (2001), após verificar ausência de efeito do supermagro sobre a produção de pimentão, cita que, mesmo sem interferir no desempenho produtivo das culturas, em solos sob manejo equilibrado, os biofertilizantes podem contribuir para elevar os teores foliares de alguns nutrientes.

O objetivo do trabalho foi estudar substratos com compostos contendo resíduos de AV e a aplicação foliar do biofertilizante supermagro na formação de mudas de maracujazeiro-amarelo.

\section{Material e Métodos}

O experimento foi realizado de dezembro de 2009 a fevereiro de 2010, em casa de vegetação do Departamento de Agronomia da UNICENTRO, Campus CEDETEG, município de GuarapuavaPR, localizado nas coordenadas $25^{\circ} 23^{\prime} \mathrm{S}, 51^{\circ} 29^{\prime} \mathrm{W}$ e $1.043 \mathrm{~m}$ (IBGE, 2009), onde o clima é tipo Cfb (Köppen), com verão ameno, geadas no inverno e precipitação anual de 1.800 a $2.000 \mathrm{~mm}$ (IAPAR, 2000). O solo foi coletado no campo experimental do Campus, na camada de 0,2-0,4 m de um Latossolo Bruno argiloso, cuja caracterização química inicial revelou: $\mathrm{V}: 21 \% ; \mathrm{pH}_{\mathrm{CaCl}_{2}}$ : 4,8; $\mathrm{MO}: 29 \mathrm{~g} \mathrm{dm}^{-3}$; P(Mehlich): $0,70 \mathrm{mg} \mathrm{dm}^{-3} ; \mathrm{Al}^{3+}: 0,02, \mathrm{Ca}^{2+}: 0,92$, $\mathrm{Mg}^{2+}: 0,65 \mathrm{e} \mathrm{K}^{+}: 0,10 \mathrm{cmol}_{\mathrm{c}} \mathrm{dm}^{-3}$. Após secagem e peneiramento, o solo foi incubado por 50 dias com calcário (CaO-28\%, MgO-20\%, PRNT-80\%), na dose calculada para atingir $\mathrm{V}=70 \%$.

O delineamento foi de blocos ao acaso com parcelas subdivididas e quatro repetições. Nas parcelas estudou-se aplicação e não aplicação foliar do supermagro, elaborado com a mistura inicial de $200 \mathrm{~L}$ de água com $20 \mathrm{~L}$ de esterco bovino, mais 
adições de $1 \mathrm{~kg}$ a cada 3 dias da mistura de sais adquirida como kit supermagro Natural Rural ${ }^{\circledR}(1,5$ $\mathrm{kg}$ cal hidratada; $1,0 \mathrm{~kg}$ ácido bórico, $100 \mathrm{~g}$ sulfato de ferro, $2 \mathrm{~kg}$ sulfato de magnésio, $200 \mathrm{~g}$ farinha de osso, $2 \mathrm{~kg}$ sulfato de zinco, $2 \mathrm{~kg}$ sulfato de cálcio, $50 \mathrm{~g}$ sulfato de cobalto, $100 \mathrm{~g}$ molibdato de sódio, $300 \mathrm{~g}$ sulfato de manganês), e da mistura proteica (1 L leite; 500 g melado; 100 g fígado avícola; 200 g calcário calcítico; 200 g fosfato de araxá; 200 g farinha de osso), fermentando por 30 dias.

Nas subparcelas estudaram-se os substratos: solo (S); solo + esterco bovino curtido ( $\mathrm{S}+\mathrm{E})$; solo + composto de esterco e aveia preta (S+CAP); solo + composto de esterco e azevém ( $\mathrm{S}+\mathrm{CAZ})$; solo + composto de esterco e nabo forrageiro ( $\mathrm{S}+\mathrm{CNF}$.); e solo + composto de esterco e ervilhaca ( $\mathrm{S}+\mathrm{CEV})$. Foram feitas pilhas de compostagem em casa de vegetação, uma só com esterco e quatro com camadas intercaladas de resíduos de adubos verdes (AV) e esterco. A proporção de massa seca de esterco e resíduos de adubos verdes (AV) nas pilhas foi 1:1, com controle diário da temperatura por revolvimento e irrigação, até sua estabilização, considerada como final do processo de compostagem.

Sacos de polietileno $\left(1,25 \mathrm{dm}^{3}\right)$ foram preenchidos com o solo corrigido, solo misturado ao esterco curtido ou aos compostos de $\mathrm{AV}$, na proporção 1:1 (v:v). Cada saco recebeu três sementes comerciais de maracujá-amarelo (cultivar Redondo Amarelo - ISLA $^{\circledR}$ 159), permanecendo sob irrigação diária e nebulização intermitente. $\mathrm{O}$ desbaste foi aos 10 dias após a emergência (DAE), preservando-se a planta mais vigorosa. Nesta data, fez-se a primeira adubação foliar (pulverização até o gotejamento) com 1,67\% de supermagro na calda. Aos 25 e 40 DAE foram feitas mais duas adubações, com $3,33 \%$ e $5,00 \%$, respectivamente.

As plantas foram colhidas aos 50 DAE, avaliando-se a altura de planta (AP), do colo ao ápice, o diâmetro do caule (DC), na altura do colo, a massa fresca total (MFT), massa seca de parte aérea (MSPA), de raízes (MSR) e total (MST), porcentagem de massa seca $[\% \mathrm{MS}=100-(\mathrm{MFT}-$ MST/MFT)x100], relação parte aérea/raízes (PA/R = MSPA/MSR), área foliar (AF), obtida por imagem digital das folhas (plano único) e processamento no programa Image $J^{\circledR}$ (ABRAMOFF; MAGALHÃES; RAM, 2004), volume radicular (VR), obtido pela submersão das raízes em proveta graduada, e extensão radicular (ER), obtida por imagem digital das raízes e processamento no programa Image ${ }^{\circledR}$.

Após as determinações com o material fresco, enxaguou-se a parte aérea (PA) e as raízes (SR) de cada muda em solução com detergente neutro (1\%) e depois em água destilada, seguindo-se a secagem em estufa de circulação forçada de ar a $65^{\circ} \mathrm{C}$ por 72 horas. O material seco foi pesado e, juntandose PA + SR, moído (Wiley) para análise dos teores de $\mathrm{N}, \mathrm{P}, \mathrm{K}, \mathrm{Ca}, \mathrm{Mg}, \mathrm{Cu}, \mathrm{Mn}$ e Zn, no Laboratório de Solos e Nutrição de Plantas da UNICENTRO, conforme metodologia descrita em EMBRAPA (2009). Os substratos e o biofertilizante foram analisados no Laboratório de Solos e Fertilizantes do Departamento de Ciência do Solo da UNESP, em Botucatu-SP, conforme Raij et al. (2001) e EMBRAPA (2009), respectivamente.

Os dados foram submetidos à análise de variância e, em caso de significância, as médias foram comparadas pelo teste de Tukey $(\alpha=0,05)$, utilizando-se o programa estatístico ASSISTAT (SILVA, 2011).

\section{Resultados e Discussão}

Os dados da análise química do supermagro (Tabela 1) revelaram que o biofertilizante elaborado apresentou teores de N, P e K similares, teores de Ca e $\mathrm{Mg}$ maiores e teores de micronutrientes menores em comparação àqueles encontrados por Rodrigues et al. (2009). Tal fato pode ser devido à variação existente dos procedimentos adotados para a fabricação do biofertilizante (BETTIOL; TRATCH; GALVÃO, 1997), bem como à composição química variável dos estercos e dos sais dos kits comercializados de supermagro. 
Tabela 1. Composição química do biofertilizante supermagro utilizado no experimento*. Guarapuava-PR, 2010.

\begin{tabular}{|c|c|c|c|c|c|c|c|c|}
\hline $\mathrm{pH}$ & $\mathrm{N}$ & $\mathrm{P}$ & $\mathrm{K}$ & $\mathrm{Ca}$ & $\mathrm{Mg}$ & $\mathrm{Cu}$ & $\mathrm{Mn}$ & $\mathrm{Zn}$ \\
\hline $\mathrm{H}_{2} \mathrm{O}$ & & ----- & $\mathrm{g} \mathrm{L}^{-1}$ & - & & ---- & $\mathrm{mg} \mathrm{L}^{-1}$ & ---- \\
\hline 5,60 & 1,54 & 0,15 & 2,99 & 2,65 & 1,30 & 80 & 160 & 143 \\
\hline
\end{tabular}

*Conforme metodologia descrita em EMBRAPA (2009).

Fonte: Elaboração dos autores.

No presente caso, os teores de macronutrientes mais elevados foram os de $\mathrm{K}$ e $\mathrm{Ca}$, sendo os de $\mathrm{P}$ os mais baixos. Quanto aos micronutrientes, os teores de $\mathrm{Cu}$ foram os mais baixos, sendo os teores de $\mathrm{Mn}$ e $\mathrm{Zn}$ quase equivalentes entre si. A acidez $(\mathrm{pH}$ $=5,6)$ do biofertilizante mostrou não ser limitante para a formulação de caldas e para a absorção dos cátions, que é maior em valores próximos de 6,0 (MALAVOLTA, 1980). Segundo Camargo (1970), que estudou a aplicação foliar de $\mathrm{N}$ (fornecido como uréia), a maior intensidade de absorção do elemento ocorreu em pH 5 a 8.

Os resultados das análises químicas dos substratos encontram-se na Tabela 2. As combinações de solo com esterco (S+E) e solo com compostos de AV $(\mathrm{S}+\mathrm{CAP}, \mathrm{S}+\mathrm{CAZ}, \mathrm{S}+\mathrm{CNF}, \mathrm{S}+\mathrm{CEV})$ apresentaram teores de MO e nutrientes e valores de CTC maiores em relação ao solo (S) isoladamente, confirmando que os resíduos orgânicos são fontes alternativas importantes de nutrientes, e justificando as recomendações de adição de $\mathrm{MO}$ aos substratos para a formação de mudas de qualidade (COSTA et al., 2008).

Tabela 2. Composição química dos substratos utilizados no experimento*. Guarapuava-PR, 2010.

\begin{tabular}{lccccccc}
\hline \multirow{2}{*}{ Indicador químico } & \multicolumn{7}{c}{ Substratos } \\
\cline { 2 - 7 } & & $\mathrm{S}^{1}$ & $\mathrm{~S}+\mathrm{E}$ & $\mathrm{S}+\mathrm{CAP}$ & $\mathrm{S}+\mathrm{CAZ}$ & $\mathrm{S}+\mathrm{CNF}$ & $\mathrm{S}+\mathrm{CEV}$ \\
\hline $\mathrm{pH}\left(\mathrm{CaCl}_{2}\right)$ & -- & 5,9 & 5,5 & 6,1 & 5,4 & 5,7 & 5,6 \\
$\mathrm{M} . \mathrm{O}$. & $\mathrm{g} \mathrm{dm}^{-3}$ & 34 & 52 & 53 & 59 & 56 & 61 \\
$\mathrm{P}_{\mathrm{resina}}$ & $\mathrm{mg} \mathrm{dm}^{-3}$ & 4 & 150 & 60 & 52 & 74 & 74 \\
$\mathrm{Al}^{+3}$ & $\mathrm{cmol}_{\mathrm{c}} \mathrm{dm}^{-3}$ & 0,1 & 0,0 & 0,1 & 0,0 & 0,1 & 0,2 \\
$\mathrm{H}+\mathrm{Al}$ & $\mathrm{cmol}_{\mathrm{c}} \mathrm{dm}^{-3}$ & 2,5 & 3,0 & 2,3 & 3,4 & 2,9 & 3,6 \\
$\mathrm{~K}^{+}$ & $\mathrm{cmol}_{\mathrm{c}} \mathrm{dm}^{-3}$ & 0,07 & 1,2 & 0,7 & 1,1 & 0,8 & 1,8 \\
$\mathrm{Ca}^{+2}$ & $\mathrm{cmol}_{\mathrm{c}} \mathrm{dm}^{-3}$ & 3,2 & 4,9 & 6,4 & 4,2 & 5,0 & 4,2 \\
$\mathrm{Mg}^{+2}$ & $\mathrm{cmol}_{\mathrm{c}} \mathrm{dm}^{-3}$ & 2,3 & 4,9 & 2,9 & 2,8 & 2,6 & 2,9 \\
$\mathrm{SB}$ & $\mathrm{cmol}_{\mathrm{c}} \mathrm{dm}^{-3}$ & 5,5 & 11,0 & 10,0 & 8,1 & 8,5 & 8,9 \\
$\mathrm{CTC}$ & $\mathrm{cmol}_{\mathrm{c}} \mathrm{dm}^{-3}$ & 8,0 & 14,0 & 12,3 & 11,4 & 11,4 & 12,5 \\
$\mathrm{~V}$ & $\%_{0}$ & 69 & 79 & 81 & 71 & 75 & 71 \\
$\mathrm{Cu}$ & 1,2 & 2 & 1,4 & 1,5 & 1,5 & 1,7 \\
$\mathrm{Mn}^{+2}$ & $\mathrm{mg} \mathrm{dm}^{-3}$ & 1,1 & 2 & 1,7 & 1,7 & 1,9 & 3,5 \\
$\mathrm{Zn}^{+2}$ & $\mathrm{mg} \mathrm{dm}^{-3}$ & 0,8 & 11,9 & 7,5 & 5,5 & 7,3 & 8,2 \\
\hline
\end{tabular}

${ }^{1} \mathrm{~S}=$ solo; $\mathrm{S}+\mathrm{E}=$ solo + esterco bovino curtido; $\mathrm{S}+\mathrm{CAP}=$ solo + composto de aveia preta, $\mathrm{S}+\mathrm{CAZ}=$ solo + composto de azevém, $\mathrm{S}+\mathrm{CNF}=$ solo + composto de nabo forrageiro, $\mathrm{S}+\mathrm{CEV}=$ solo + composto de ervilhaca. ${ }^{*}$ Conforme Raij et al. (2001).

Fonte: Elaboração dos autores.

Os resultados da Tabela 2 também revelam que a calagem, na dose calculada para $\mathrm{V}=70 \%$, e que o período de incubação de 50 dias, foram ambos eficientes na correção da acidez do solo, uma vez que o tratamento $\mathrm{S}$ apresentou $\mathrm{V}=69 \%$. Quanto a este indicador químico do solo, os tratamentos $\mathrm{S}+\mathrm{E}$ 
e $\mathrm{S}+\mathrm{CAP}$ se sobressaíram aos demais, com $\mathrm{V}=79$ e $81 \%$, respectivamente. O substrato $\mathrm{S}+\mathrm{E}$ também apresentou maiores teores de $\mathrm{P}, \mathrm{Mg}, \mathrm{Cu}$ e $\mathrm{Zn}$, enquanto o substrato $\mathrm{S}+\mathrm{CAP}$ apresentou os maiores valores para $\mathrm{pH}$ e $\mathrm{Ca}$. Já o substrato $\mathrm{S}+\mathrm{CEV}$ obteve os maiores teores de $\mathrm{Mn}$ e de $\mathrm{K}$, cujos teores em todos os substratos com resíduos orgânicos $(0,7-1,8$ $\mathrm{cmol}_{\mathrm{c}} \mathrm{dm}^{-3}$ ) foram muito superiores em relação ao solo isoladamente $\left(0,07 \mathrm{cmol}_{\mathrm{c}} \mathrm{dm}^{-3}\right)$.

Quanto ao crescimento das mudas, houve efeito isolado de substrato para $\mathrm{DC}$ e interação entre substrato e adubação foliar para AP, AF, ER e VR (Tabela 3). As mudas apresentaram DC superior com o substrato $\mathrm{S}+\mathrm{E}$ em relação aos substratos com compostos de adubos verdes, que por sua vez superaram o resultado obtido com solo isolado (S). As plantas crescidas no tratamento formado por solo (S) apresentaram os piores resultados, indo ao encontro dos dados obtidos por Silva et al. (2010), resultado que está em concordância com a menor fertilidade química do substrato em relação aos demais tratamentos (Tabela 2). Serrano et al. (2006), estudando a produção de mudas de maracujazeiro-amarelo em substratos alternativos observou melhor estado nutricional e crescimento nos tratamentos: bagaço de cana + torta de filtro $(3: 2 ; \mathrm{v}: \mathrm{v})+7,3 \mathrm{~kg} \mathrm{~m}^{-3}$ de Osmocote $^{\circledR}$ (14-14-14) e Plantmax ${ }^{\circledR}+7,3 \mathrm{~kg} \mathrm{~m}^{-3}$ de Osmocote ${ }^{\circledR}$, os quais apresentaram, 60 dias após a semeadura, alturas médias de 62,84 e $56,30 \mathrm{~cm}$ respectivamente. Esses valores se aproximam aos encontrados nos tratamentos S+CAP com supermagro e S+CNF sem o biofertilizante (Tabela 3).

Nas demais variáveis de crescimento, S+E manteve os melhores resultados, como verificado por Mendonça et al. (2009), que obteve melhor desempenho de mudas de maracujazeiro-amarelo crescidas em substrato solo + esterco bovino (3:1; v:v) do que nos demais, sendo o substrato solo o de pior performance. A explicação para esta resultado pode estar nas melhores condições de fertilidade, com destaque para V\% e para os teores de $\mathrm{Cu}, \mathrm{Zn}$ e, sobretudo, de $\mathrm{P}$ e $\mathrm{Mg}$, macronutrientes cujos papéis energético e na formação da clorofila, respectivamente, são fundamentais para absorver outros nutrientes e transformar energia luminosa em compostos de carbono para o crescimento das plantas (MARSCHNER, 1995). O melhor enraizamento obtido no substrato $\mathrm{S}+\mathrm{E}$, pelos resultados de ER e VR, também garante maior aproveitamento dos nutrientes. Boechat et al. (2010), trabalhando com duas cultivares de maracujazeiro-amarelo, testaram substrato plantmax ${ }^{\circledR}$ e as alternativas esterco + terra de barranco (3:1) e substrato a base de fibra de coco, encontrando melhores resultados de AP e DC com plantmax ${ }^{\circledR}$ e com esterco + terra, confirmando que substratos feitos pelos produtores com resíduos orgânicos podem ter desempenho similar aos substratos industrializados.

A aplicação foliar do biofertilizante não teve efeito ou teve efeito variável no crescimento das mudas. Combinada ao substrato $\mathrm{S}+\mathrm{CAP}$, mesmo sem aumento significativo nas variáveis de raiz, o supermagro aumentou significativamente AP e AF na parte aérea, sendo estes resultados superados somente pelos do substrato $\mathrm{S}+\mathrm{E}$. Já em combinação ao substrato $\mathrm{S}+\mathrm{CNF}$, o biofertilizante teve efeito negativo em todas as variáveis. No geral, as mudas tiveram melhor crescimento de parte aérea em $\mathrm{S}+\mathrm{E}$ sem supermagro via foliar, seguido de S+CAP com supermagro via foliar. Estudando o crescimento vegetativo do maracujazeiro-amarelo, Costa et al. (2008) não encontraram efeito significativo de diferentes tipos e dosagens de biofertilizantes sobre AP, DC e número de ramos terciários, mas concluíram que o crescimento foi beneficiado com 600 a $900 \mathrm{ml} /$ planta/aplicação e prejudicado com mais de $900 \mathrm{ml} /$ planta/aplicação. 
Tabela 3. Diâmetro de caule (DC), altura de planta (AP), área foliar (AF), extensão (ER) e volume radicular (VR) de mudas de maracujazeiro-amarelo aos 50 dias após a emergência, em função de substratos e adubação foliar com supermagro. Guarapuava-PR, 2010.

\begin{tabular}{ccccccccc}
\hline Indicadores/ & \multicolumn{7}{c}{ Substratos } \\
\cline { 3 - 8 } Adub. Foliar & \multicolumn{1}{c}{$\mathrm{S}^{1}$} & $\mathrm{~S}+\mathrm{E}$ & $\mathrm{S}+\mathrm{CAP}$ & $\mathrm{S}+\mathrm{CAZ}$ & $\mathrm{S}+\mathrm{CNF}$ & $\mathrm{S}+\mathrm{CEV}$ & média \\
\hline \multirow{2}{*}{$\mathrm{DC}$} & $\mathrm{S} / \mathrm{SM}^{2}$ & $1,43 \mathrm{~ns}$ & 5,53 & 4,49 & 3,98 & 4,35 & 3,80 & $3,933^{\mathrm{ns}}$ \\
$(\mathrm{mm})$ & $\mathrm{C} / \mathrm{SM}$ & 1,46 & 5,27 & 4,27 & 3,74 & 3,45 & 4,02 & 3,70 \\
& média & $1,44 \mathrm{c}^{3}$ & $5,40 \mathrm{a}$ & $4,38 \mathrm{~b}$ & $3,86 \mathrm{~b}$ & $3,90 \mathrm{~b}$ & $3,91 \mathrm{~b}$ & \\
& & & & & & & \\
$\mathrm{AP}$ & $\mathrm{S} / \mathrm{SM}$ & $4,40 \mathrm{eA}$ & $98,85 \mathrm{aA}$ & $42,15 \mathrm{bcB}$ & $37,20 \mathrm{cdA}$ & $53,85 \mathrm{bA}$ & $28,83 \mathrm{dA}$ & 44,38 \\
$(\mathrm{~cm})$ & $\mathrm{C} / \mathrm{SM}$ & $4,88 \mathrm{dA}$ & $81,38 \mathrm{aB}$ & $62,88 \mathrm{bA}$ & $29,65 \mathrm{cA}$ & $20,28 \mathrm{cB}$ & $21,88 \mathrm{cA}$ & 36,82 \\
& média & 4,64 & 90,61 & 52,51 & 33,43 & 37,06 & 25,35 & \\
& & & & & & & \\
$\mathrm{AF}$ & $\mathrm{S} / \mathrm{SM}$ & $10,3 \mathrm{dA}$ & $966,3 \mathrm{aA}$ & $514,6 \mathrm{bcB}$ & $435,4 \mathrm{cA}$ & $544,7 \mathrm{bA}$ & $438,1 \mathrm{cA}$ & 484,9 \\
$\left(\mathrm{~cm}{ }^{2}\right)$ & $\mathrm{C} / \mathrm{SM}$ & $24,2 \mathrm{eA}$ & $763,6 \mathrm{aB}$ & $630,0 \mathrm{bA}$ & $473,1 \mathrm{cA}$ & $354,3 \mathrm{~dB}$ & $443,2 \mathrm{cdA}$ & 448,1 \\
& média & 17,2 & 864,9 & 572,5 & 454,2 & 449,5 & 440,7 & \\
& & & & & & \\
$\mathrm{ER}$ & $\mathrm{S} / \mathrm{SM}$ & $247 \mathrm{dA}$ & $8945 \mathrm{aA}$ & $5867 \mathrm{bcA}$ & $6093 \mathrm{bA}$ & $5566 \mathrm{bcA}$ & $4959 \mathrm{cA}$ & 5280 \\
$(\mathrm{~cm})$ & $\mathrm{C} / \mathrm{SM}$ & $311 \mathrm{dA}$ & $9442 \mathrm{aA}$ & $5871 \mathrm{bA}$ & $4297 \mathrm{cB}$ & $4288 \mathrm{cB}$ & $5018 \mathrm{bcA}$ & 4871 \\
& média & 279 & 9193 & 5869 & 5195 & 4927 & 4989 & \\
& & & & & & & \\
$\mathrm{VR}$ & $\mathrm{S} / \mathrm{SM}$ & $1,25 \mathrm{cA}$ & $14,75 \mathrm{aB}$ & $9,00 \mathrm{bA}$ & $9,25 \mathrm{bA}$ & $11,50 \mathrm{bA}$ & $9,25 \mathrm{bA}$ & 9,17 \\
$\left(\mathrm{~cm}{ }^{3}\right)$ & $\mathrm{C} / \mathrm{SM}$ & $1,00 \mathrm{cA}$ & $17,00 \mathrm{aA}$ & $10,75 \mathrm{bA}$ & $8,00 \mathrm{bA}$ & $7,75 \mathrm{bB}$ & $9,25 \mathrm{bA}$ & 8,96 \\
& média & 1,13 & 15,87 & 9,88 & 8,62 & 9,63 & 9,25 & \\
\hline
\end{tabular}

${ }^{1} \mathrm{~S}=$ solo $; \mathrm{S}+\mathrm{E}=$ solo + esterco bovino curtido; $\mathrm{S}+\mathrm{CAP}=$ solo + composto de aveia preta, $\mathrm{S}+\mathrm{CAZ}=$ solo + composto de azevém, $\mathrm{S}+\mathrm{CNF}=$ solo + composto de nabo forrageiro, $\mathrm{S}+\mathrm{CEV}=$ solo + composto de ervilhaca.

${ }^{2} \mathrm{~S} / \mathrm{SM}=$ sem adubação foliar de supermagro; $\mathrm{C} / \mathrm{SM}=$ com adubação foliar de supermagro;

${ }^{\text {ns } N a ̃ o ~ s i g n i f i c a t i v o ~ a o ~ n i ́ v e l ~ d e ~ 5 \% ~ d e ~ p r o b a b i l i d a d e ~ n a ~ a n a ́ l i s e ~ d e ~ v a r i a ̂ n c i a . ~}$

${ }^{3}$ Letras minúsculas na linha e maiúsculas na coluna comparam as médias pelo teste de Tukey a $5 \%$ de probabilidade, por variável.

Fonte: Elaboração dos autores.

Quanto à massa seca das plantas (Tabela 4), houve interação entre substrato e adubação foliar, com exceção à \%MS, que mostrou efeito somente de substrato, novamente evidenciando a superioridade de $\mathrm{S}+\mathrm{E}$ sobre os demais, com significância na comparação a $\mathrm{S}+\mathrm{CNF}, \mathrm{S}+\mathrm{CAZ}$ e $\mathrm{S}+\mathrm{CAP}$. Nos resultados de MSPA, MSR, MST, o substrato $\mathrm{S}+\mathrm{E}$ manteve desempenho superior aos substratos com compostos de $\mathrm{AV}$, que também mantiveram-se superiores ao solo isolado (S) como substrato. Entre os substratos com compostos de AV, $\mathrm{S}+\mathrm{CAP}$ manteve-se beneficiado pelo biofertilizante e, combinado a ele, obteve os melhores resultados, inferiores apenas em comparação a S+E. Sem aplicação do supermagro, $\mathrm{S}+\mathrm{CNF}$ repetiu o bom desempenho observado nos indicadores de crescimento, sendo significativamente superior a S+CAZ para MSPA e MST.

Houve, também, interação entre substrato e adubação foliar no resultados de relação PA/R (Tabela 4). Sem o biofertilizante via foliar, $\mathrm{S}+\mathrm{E}$ gerou PA/R superior à dos demais substratos, equivalentes entre si, mas quando o supermagro foi aplicado, a relação PA/R das mudas diminuiu em $\mathrm{S}+\mathrm{E}$, igualando-o aos demais substratos, e com uso do supermagro os substratos S+CAP e $\mathrm{S}+\mathrm{CAZ}$ superaram os demais, com significância na comparação a $\mathrm{S}+\mathrm{CNF}$. 
Tabela 4. Massa seca de parte aérea (MSPA), raízes (MSR) e total (MST), relação parte aérea/raiz (PA/R) e porcentagem de matéria seca (\%MS) de mudas de maracujazeiro-amarelo aos 50 dias após a emergência, em função de substratos e adubação foliar com supermagro. Guarapuava-PR, 2010.

\begin{tabular}{|c|c|c|c|c|c|c|c|c|}
\hline \multirow{2}{*}{\multicolumn{2}{|c|}{$\begin{array}{l}\text { Indicadores/ } \\
\text { Adub. Foliar }\end{array}$}} & \multicolumn{7}{|c|}{ Substratos } \\
\hline & & $\mathrm{S}^{1}$ & $\mathrm{~S}+\mathrm{E}$ & $\mathrm{S}+\mathrm{CAP}$ & $\mathrm{S}+\mathrm{CAZ}$ & $\mathrm{S}+\mathrm{CNF}$ & $\mathrm{S}+\mathrm{CEV}$ & média \\
\hline \multirow{3}{*}{$\begin{array}{l}\text { MSPA } \\
(\mathrm{g})\end{array}$} & ${\mathrm{S} / \mathrm{SM}^{2}}^{2}$ & $0,07 \mathrm{dA}^{3}$ & $6,94 \mathrm{aA}$ & $2,58 \mathrm{bcB}$ & $2,31 \mathrm{cA}$ & $3,06 \mathrm{bA}$ & $2,45 \mathrm{bcA}$ & 2,90 \\
\hline & $\mathrm{C} / \mathrm{SM}$ & $0,12 \mathrm{eA}$ & $5,53 \mathrm{aB}$ & $3,79 \mathrm{bA}$ & $2,19 \mathrm{cdA}$ & $1,58 \mathrm{~dB}$ & $2,37 \mathrm{cA}$ & 2,59 \\
\hline & média & 0,10 & 6,24 & 3,18 & 2,25 & 2,35 & 2,41 & \\
\hline \multirow{3}{*}{$\begin{array}{c}\text { MSR } \\
(\mathrm{g})\end{array}$} & $\mathrm{S} / \mathrm{SM}$ & $0,03 \mathrm{cA}$ & $1,20 \mathrm{aB}$ & $0,77 \mathrm{bA}$ & $0,66 \mathrm{bA}$ & $0,81 \mathrm{bA}$ & $0,77 \mathrm{bA}$ & 0,70 \\
\hline & $\mathrm{C} / \mathrm{SM}$ & $0,04 \mathrm{eA}$ & $1,32 \mathrm{aA}$ & $0,79 \mathrm{bA}$ & $0,47 \mathrm{~dB}$ & $0,55 \mathrm{cdB}$ & $0,69 \mathrm{bcA}$ & 0,64 \\
\hline & média & 0,03 & 1,26 & 0,78 & 0,56 & 0,68 & 0,73 & \\
\hline \multirow{3}{*}{$\begin{array}{c}\text { MST } \\
(\mathrm{g})\end{array}$} & $\mathrm{S} / \mathrm{SM}$ & $0,10 \mathrm{dA}$ & $8,14 \mathrm{aA}$ & $3,35 \mathrm{bcB}$ & $2,96 \mathrm{cA}$ & $3,86 \mathrm{bA}$ & $3,22 \mathrm{bcA}$ & 3,61 \\
\hline & $\mathrm{C} / \mathrm{SM}$ & $0,16 \mathrm{eA}$ & $6,85 \mathrm{aB}$ & $4,58 \mathrm{bA}$ & $2,66 \mathrm{cdA}$ & $2,12 \mathrm{~dB}$ & $3,06 \mathrm{cA}$ & 3,24 \\
\hline & média & 0,13 & 7,50 & 3,96 & 2,81 & 2,99 & 3,14 & \\
\hline \multirow{3}{*}{$\mathrm{PA} / \mathrm{R}$} & $\mathrm{S} / \mathrm{SM}$ & $2,50 \mathrm{bA}$ & $5,85 \mathrm{aA}$ & $3,38 \mathrm{bB}$ & $3,50 \mathrm{bB}$ & $3,86 \mathrm{bA}$ & $3,20 \mathrm{bA}$ & $3,72^{\text {ns }}$ \\
\hline & $\mathrm{C} / \mathrm{SM}$ & $3,29 \mathrm{abA}$ & $4,18 \mathrm{abB}$ & $4,78 \mathrm{aA}$ & $4,73 \mathrm{aA}$ & $2,97 \mathrm{bA}$ & $3,44 \mathrm{abA}$ & 3,90 \\
\hline & média & 2,89 & 5,02 & 4,08 & 4,12 & 3,42 & 3,32 & \\
\hline \multirow{3}{*}{$\begin{array}{l}\text { MS } \\
(\%)\end{array}$} & $\mathrm{S} / \mathrm{SM}$ & $15,8^{\mathrm{ns}}$ & 16,8 & 12,8 & 13,8 & 13,6 & 15,2 & $14,7^{\text {ns }}$ \\
\hline & $\mathrm{C} / \mathrm{SM}$ & 14,8 & 15,8 & 14,1 & 13,9 & 13,1 & 15,5 & 14,5 \\
\hline & média & $15,3 \mathrm{ab}$ & $16,3 \mathrm{a}$ & $13,5 b$ & $13,8 \mathrm{~b}$ & $13,3 b$ & $15,3 \mathrm{ab}$ & \\
\hline
\end{tabular}

${ }^{1} \mathrm{~S}=$ solo; $\mathrm{S}+\mathrm{E}=$ solo + esterco bovino curtido; $\mathrm{S}+\mathrm{CAP}=$ solo + composto de aveia preta, $\mathrm{S}+\mathrm{CAZ}=$ solo + composto de azevém, $\mathrm{S}+\mathrm{CNF}=$ solo + composto de nabo forrageiro, $\mathrm{S}+\mathrm{CEV}=$ solo + composto de ervilhaca.

${ }^{2} \mathrm{~S} / \mathrm{SM}=$ sem adubação foliar de supermagro; $\mathrm{C} / \mathrm{SM}=$ com adubação foliar de supermagro;

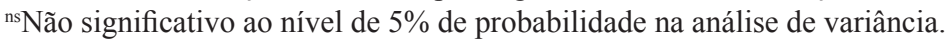

${ }^{3}$ Letras minúsculas na linha e maiúsculas na coluna comparam as médias pelo teste de Tukey a 5\% de probabilidade, por variável.

Fonte: Elaboração dos autores.

Nos resultados de acúmulo de nutrientes na MST das mudas aos 50 DAE (Tabela 5), não aparecem os dados relativos ao substrato $\mathrm{S}$ que, com o pequeno acúmulo de biomassa, não apresentaram material suficiente para análise. Houve efeito isolado de substrato e adubação foliar para o acúmulo de N, sendo as mudas adubadas com supermagro as de menor acúmulo de $\mathrm{N}$, o que é condizente com a diminuição de MST das mudas em dois substratos ( $\mathrm{S}+\mathrm{E}$ e $\mathrm{S}+\mathrm{CNF})$ e aumento em apenas um substrato (S+CAP) com o biofertilizante, diminuindo a média geral. Quanto aos substratos, as mudas de $\mathrm{S}+\mathrm{E}$ mostraram maior acúmulo de $\mathrm{N}$, em acordo com o maior acúmulo de MST (Tabela 4), e embora $\mathrm{S}+\mathrm{CAP}$ tenha apresentado bons resultados de MST sob uso de supermagro, o acúmulo de $\mathrm{N}$ foi inferior ao dos demais substratos na média dos níveis de adubação foliar, com significância na comparação a $\mathrm{S}+\mathrm{CAZ}$, que se destacou no acúmulo de $\mathrm{N}$.

O substrato $\mathrm{S}+\mathrm{CAZ}$, apesar de utilizar resíduo de gramínea, cuja relação $\mathrm{C} / \mathrm{N}$ é reconhecidamente mais alta (POTT; MÜLLER; BERTELLI, 2007) que das leguminosas $(\mathrm{S}+\mathrm{CEV})$ e brássicas $(\mathrm{C}+\mathrm{CNF})$, resultou na segunda maior extração de N. Este resultado pode ser devido ao fato deste adubo verde ser mais tardio que as demais espécies (FLARESSO; GROSS; ALMEIDA, 2001; POSTIGLIONI, 1982), não alcançando valores tão elevados quanto os esperados para a relação $\mathrm{C} / \mathrm{N}$ na época de corte das plantas no campo. Ademais, mesmo com a compostagem em casa de vegetação, houve 
irrigação das pilhas para controle da temperatura, e $\mathrm{N}$ mais intensamente durante a decomposição dos a baixa relação $\mathrm{C} / \mathrm{N}$ característica das leguminosas resíduos, potencializando a lixiviação e perda do $\mathrm{N}$ $(\mathrm{S}+\mathrm{CEV})$ e brássicas $(\mathrm{C}+\mathrm{CNF})$ pode ter liberado pela água de irrigação.

Tabela 5. Acúmulo N, $\mathrm{P}, \mathrm{K}, \mathrm{Ca}, \mathrm{Mg}\left(\mathrm{x} 10^{-2} \mathrm{~g} \mathrm{planta}^{-1}\right) \mathrm{Zn}, \mathrm{Cu}$ e $\mathrm{Mn}\left(\mathrm{x} 10^{-2} \mathrm{mg}\right.$ planta $\left.{ }^{-1}\right)$ na massa seca total de mudas de maracujazeiro-amarelo aos 50 dias após a emergência, em função de substratos e adubação foliar com supermagro. Guarapuava-PR, 2010.

\begin{tabular}{|c|c|c|c|c|c|c|c|}
\hline \multirow{2}{*}{\multicolumn{2}{|c|}{$\begin{array}{l}\text { Nutrientes / } \\
\text { Adub. foliar }\end{array}$}} & \multicolumn{6}{|c|}{ Substratos } \\
\hline & & $\mathrm{S}+\mathrm{E}^{1}$ & $\mathrm{~S}+\mathrm{CAP}$ & $\mathrm{S}+\mathrm{CAZ}$ & $\mathrm{S}+\mathrm{CNF}$ & $\mathrm{S}+\mathrm{CEV}$ & média \\
\hline \multirow{3}{*}{$\mathrm{N}$} & $\mathrm{S} / \mathrm{SM}^{2}$ & 3,71 & 0,99 & 2,35 & 1,65 & 1,00 & $1,94 \mathrm{~A}$ \\
\hline & $\mathrm{C} / \mathrm{SM}$ & 3,32 & 0,94 & 1,09 & 0,63 & 1,04 & $1,40 \mathrm{~B}$ \\
\hline & média & $3,51 \mathrm{a}^{3}$ & $0,97 \mathrm{c}$ & $1,72 \mathrm{~b}$ & $1,14 \mathrm{bc}$ & $1,02 \mathrm{c}$ & \\
\hline \multirow{3}{*}{$\mathrm{P}$} & $\mathrm{S} / \mathrm{SM}$ & $0,10 \mathrm{aA}$ & $0,03 \mathrm{cB}$ & $0,05 \mathrm{bcA}$ & $0,10 \mathrm{aA}$ & $0,07 \mathrm{bB}$ & $0,07^{\mathrm{ns}}$ \\
\hline & $\mathrm{C} / \mathrm{SM}$ & $0,10 \mathrm{aA}$ & $0,06 \mathrm{bA}$ & $0,06 \mathrm{bA}$ & $0,06 \mathrm{bB}$ & $0,11 \mathrm{aA}$ & 0,08 \\
\hline & média & 0,10 & 0,04 & 0,06 & 0,08 & 0,09 & \\
\hline \multirow{3}{*}{$\mathrm{K}$} & $\mathrm{S} / \mathrm{SM}$ & $26,6^{\mathrm{ns}}$ & 13,1 & 10,5 & 12,8 & 10,6 & $14,7^{\mathrm{ns}}$ \\
\hline & $\mathrm{C} / \mathrm{SM}$ & 20,6 & 16,0 & 9,4 & 7,3 & 9,3 & 12,5 \\
\hline & média & $23,6 \mathrm{a}$ & $14,5 \mathrm{~b}$ & $9,9 \mathrm{c}$ & $10,0 \mathrm{c}$ & $9,9 \mathrm{c}$ & \\
\hline \multirow{3}{*}{$\mathrm{Ca}$} & $\mathrm{S} / \mathrm{SM}$ & $7,74 \mathrm{aA}$ & $3,79 \mathrm{bA}$ & $3,95 \mathrm{bA}$ & $6,43 \mathrm{aA}$ & $4,33 \mathrm{bA}$ & $5,25 \mathrm{~ns}$ \\
\hline & $\mathrm{C} / \mathrm{SM}$ & $7,11 \mathrm{aA}$ & $5,18 \mathrm{abA}$ & $3,43 \mathrm{bcA}$ & $3,10 \mathrm{cB}$ & $4,38 \mathrm{bcA}$ & 4,64 \\
\hline & média & 7,42 & 4,49 & 3,69 & 4,77 & 4,36 & \\
\hline \multirow{3}{*}{$\mathrm{Mg}$} & $\mathrm{S} / \mathrm{SM}$ & 0,31 ns & 0,10 & 0,10 & 0,12 & 0,08 & $0,14 \mathrm{~ns}$ \\
\hline & $\mathrm{C} / \mathrm{SM}$ & 0,28 & 0,14 & 0,10 & 0,07 & 0,07 & 0,13 \\
\hline & média & $0,30 \mathrm{a}$ & $0,13 \mathrm{~b}$ & $0,10 \mathrm{~b}$ & $0,10 \mathrm{~b}$ & $0,08 \mathrm{~b}$ & \\
\hline \multirow{3}{*}{$\mathrm{Zn}$} & $\mathrm{S} / \mathrm{SM}$ & 51,2 ns & 26,9 & 19,2 & 26,2 & 25,2 & $29,7 \mathrm{~ns}$ \\
\hline & $\mathrm{C} / \mathrm{SM}$ & 62,8 & 29,4 & 18,2 & 16,6 & 24,6 & 30,3 \\
\hline & média & 57,0 a & $28,1 \mathrm{~b}$ & $18,7 \mathrm{~b}$ & $21,4 \mathrm{~b}$ & $24,9 \mathrm{~b}$ & \\
\hline \multirow{3}{*}{$\mathrm{Cu}$} & $\mathrm{S} / \mathrm{SM}$ & $24,6 \mathrm{aA}$ & $8,1 \mathrm{bA}$ & $5,6 \mathrm{bA}$ & $6,9 \mathrm{bA}$ & $7,0 \mathrm{bA}$ & $10,4^{\mathrm{ns}}$ \\
\hline & $\mathrm{C} / \mathrm{SM}$ & $16,2 \mathrm{aB}$ & $10,6 \mathrm{bA}$ & $4,9 \mathrm{cA}$ & $3,8 \mathrm{cA}$ & $8,0 \mathrm{bcA}$ & 8,7 \\
\hline & média & 20,4 & 9,4 & 5,2 & 5,3 & 7,5 & \\
\hline \multirow{3}{*}{$\mathrm{Mn}$} & $\mathrm{S} / \mathrm{SM}$ & $16,86^{\mathrm{ns}}$ & 12,7 & 8,0 & 9,3 & 7,7 & $10,9^{\mathrm{ns}}$ \\
\hline & $\mathrm{C} / \mathrm{SM}$ & 17,42 & 10,5 & 9,2 & 5,5 & 7,8 & 10,1 \\
\hline & média & $17,14 \mathrm{a}$ & $11,6 \mathrm{~b}$ & $8,6 \mathrm{~b}$ & $7,4 \mathrm{~b}$ & $7,8 \mathrm{~b}$ & \\
\hline
\end{tabular}

${ }^{1} \mathrm{~S}=$ solo; $\mathrm{S}+\mathrm{E}=$ solo + esterco bovino curtido; $\mathrm{S}+\mathrm{CAP}=$ solo + composto de aveia preta, $\mathrm{S}+\mathrm{CAZ}=$ solo + composto de azevém, $\mathrm{S}+\mathrm{CNF}=$ solo + composto de nabo forrageiro, $\mathrm{S}+\mathrm{CEV}=$ solo + composto de ervilhaca.

${ }^{2} \mathrm{~S} / \mathrm{SM}=$ sem adubação foliar de supermagro; $\mathrm{C} / \mathrm{SM}=$ com adubação foliar de supermagro;

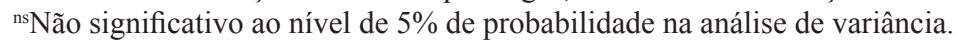

${ }^{3}$ Letras minúsculas na linha e maiúsculas na coluna comparam as médias pelo teste de Tukey a 5\% de probabilidade, por variável.

Fonte: Elaboração dos autores.

Houve interação significativa entre substrato e adubação foliar para os acúmulos de $\mathrm{P}, \mathrm{Ca}$ e $\mathrm{Cu}$, e 
efeito isolado de substrato para os acúmulos de K, $\mathrm{Mg}, \mathrm{Zn}$ e Mn na MST das mudas. No caso do P, o substrato $\mathrm{S}+\mathrm{E}$, cujo teor de $\mathrm{P}$ foi de $150 \mathrm{mg} \mathrm{dm}^{-3}$ (Tabela 2) e gerou mudas com maior MST, obteve os maiores acúmulos nas mudas, mas equivaleu-se a $\mathrm{S}+\mathrm{CNF}$ sem biofertilizante foliar e $\mathrm{S}+\mathrm{CEV}$ com biofertilizante foliar, sendo os teores de $\mathrm{P}$ destes substratos iguais a $74 \mathrm{mg} \mathrm{dm}^{-3}$, os maiores entre os que continham compostos de AV (Tabela 2). Em conformidade com as observações de crescimento (Tabela 3) e massa seca (Tabela 4), a aplicação de supermagro manteve efeito positivo sobre o acúmulo de $\mathrm{P}$ das mudas quando combinada a $\mathrm{S}+\mathrm{CAP}$, e negativo combinada a $\mathrm{S}+\mathrm{CNF}$.

Quanto ao acúmulo de $\mathrm{Ca}$ nas mudas, sem uso de supermagro o substrato $\mathrm{S}+\mathrm{CNF}$ novamente se equivaleu a $\mathrm{S}+\mathrm{E}$ e estes superaram os demais, mas como o supermagro manteve efeito negativo para $\mathrm{S}+\mathrm{CNF}$, afetando somente este substrato, o acúmulo de $\mathrm{Ca}$ nas mudas piorou, tornando-se o menor entre todos os substratos, com significância na comparação a S+CAP. Estes resultados estão em concordância com os teores de $\mathrm{Ca}$ em $\mathrm{S}+\mathrm{CNF}$ e $\mathrm{S}+\mathrm{CAP}$, os dois maiores entre todos os substratos (Tabela 2).

Somente nas mudas de $\mathrm{S}+\mathrm{E}$ o acúmulo de $\mathrm{Cu}$ foi afetado por supermagro, com diminuição significativa. Sem supermagro, o substrato $\mathrm{S}+\mathrm{E}$ superou os demais, sem diferença entre eles. Porém, com supermagro, $\mathrm{S}+\mathrm{E}$ superou $\mathrm{S}+\mathrm{CAP}$ e este superou os outros, diferindo-se significativamente de S+CAZ e S+CNF. Este desempenho bom de $\mathrm{S}+\mathrm{CAP}$ também se repetiu para $\mathrm{K}$, que se acumulou mais nas mudas deste substrato em relação aos demais substratos com compostos de AV, sendo superado somente por S+E. Este fato se justifica pelo acúmulo de MST das mudas (Tabela 4), pois o teor de $\mathrm{K}$ em $\mathrm{S}+\mathrm{CAP}$ foi baixo em relação aos outros substratos (Tabela 2).

Para $\mathrm{Mg}, \mathrm{Zn}$ e Mn, o acúmulo nas mudas (Tabela 5) apresentou o mesmo comportamento, sendo maior nas plantas cultivadas em $\mathrm{S}+\mathrm{E}$ e sem diferenças significativas entre os demais, em concordância com os maiores teores destes nutrientes encontrados em S+E (Tabela 2), à exceção do $\mathrm{Mn}$, que se apresentou em teor mais elevado no substrato $\mathrm{S}+\mathrm{CEV}$, sem, no entanto, ser compensado pelo menor acúmulo de massa seca das mudas neste substrato. No geral, as mudas de maracujazeiroamarelo apresentaram a seguinte ordem decrescente de acúmulo de nutrientes nas condições do estudo: $\mathrm{K}>\mathrm{Ca}>\mathrm{N}>\mathrm{Mg}>\mathrm{P}>\mathrm{Zn}>\mathrm{Cu}=\mathrm{Mn}$.

$\mathrm{O}$ crescimento do maracujazeiro exige farta disponibilidade de nutrientes, por ser uma planta de crescimento rápido e vigoroso, refletindo-se na importância da adubação orgânica para esta espécie (COSTA et al., 2008). Assim, o uso de solo isoladamente como substrato revelou os piores resultados de crescimento e acúmulo de nutrientes, em oposição ao uso de solo com esterco puro curtido, que propiciou, consistentemente, os melhores resultados de formação das mudas, sugerindo ser a maior disponibilidade de nutrientes neste composto o fator decisivo.

Entretanto, houve bom desempenho de crescimento das mudas (AP, AF, VR, MSPA e MST) com o substrato S+CAP, com aplicação foliar de supermagro, e com o substrato $\mathrm{S}+\mathrm{CNF}$ sem uso do biofertilizante, os quais apresentaram, dentre os substratos com compostos de AV, os maiores valores de $\mathrm{pH}$ e V\%, combinados a teores de $\mathrm{P}$ e Ca também entre os maiores. Assim, deve-se avaliar, no futuro, novas proporções entre solo e estes compostos de AV na formação dos substratos finais, pois é possível aumentar a proporção dos mesmos em relação ao solo, compensando o menor teor de nutrientes em comparação ao esterco (Tabela 2), e mesmo assim manter ainda redução de esterco em relação a seu uso isolado com solo para formar substratos, o que resolveria problemas de baixa disponibilidade e/ou custo elevado do esterco em determinadas situações.

Os resultados também mostram que o uso do biofertilizante supermagro não pode ser indiscriminado, devendo-se conhecer as situações em que é benéfico, como na combinação com o substrato $\mathrm{S}+\mathrm{CAP}$, para não ser recomendado 
erradamente, sob pena de duplo prejuízo para o produtor, com custos na elaboração do mesmo e perda de qualidade na formação das mudas.

\section{Conclusões}

A melhor formação de mudas de maracujazeiro amarelo se deu com o substrato solo mais esterco, em concordância com o maior fornecimento de nutrientes. Dentre os substratos com compostos de adubos verdes, aqueles com compostos de aveia preta e de nabo forrageiro destacaram-se dos demais.

$\mathrm{O}$ uso de biofertilizante supermagro teve resultado diverso sobre a formação das mudas, sendo benéfico quando combinado com o substrato com o composto de aveia preta, e prejudicial em combinação com os substratos solo mais esterco e solo mais composto de nabo forrageiro.

\section{Referências}

ABRAMOFF, W. S.; MAGALHÃES, P. J.; RAM, S. J. Image processing with Image J. Biophotonics International, Los Gatos, v. 11, n. 7, p. 36-42, 2004.

ANUÁRIO ESTATÍSTICO DA AGRICULTURA BRASILEIRA - AGRIANUAL. São Paulo: FNP, 2010, $520 \mathrm{p}$.

ARAÚJO, W. B. M.; ALENCAR, R. D.; MENDONÇA, V.; MEDEIROS, E. V.; ANDRADE, R. C.; ARAÚJO, R. R. Esterco caprino na composição de substratos para formação de mudas de mamoeiro. Ciência $e$ Agrotecnologia, Lavras, v. 34, n. 1, p. 68-73, 2010.

BETTIOL, W.; TRATCH, R.; GALVÃO, J. A. H. Controle de doenças de plantas com biofertilizantes. Jaguariúna: EMBRAPA-CNPMA, 1997. 22 p. (Circular técnica, 2).

BOECHAT, C. L.; TEIXEIRA, A. M.; COSTA, A. S. V.; SOUZA, A. P. S. B. Influência de substratos associados à adubação mineral sobre o crescimento inicial de duas cultivares de maracujazeiro-amarelo. Revista Caatinga, Mossoró, v. 23, n. 3, p. 19-25, 2010.

CAMARGO, P. N. Principios de nutrição foliar. São Paulo: Ceres, 1970. 117 p.

COSTA, E.; RODRIGUES, E. T.; ALVES, V. B.; SANTOS, L. C. R.; VIEIRA, L. C. R. Efeitos da ambiência, recipientes e substratos no desenvolvimento de mudas de maracujazeiro-amarelo em AquidauanaMS. Revista Brasileira de Fruticultura, Jaboticabal, v. 31, n. 1, p. 236-244, 2009.

COSTA, Z. V. B.; DINIZ NETO, P.; ANDRADE, R.; SANTOS, J. G. R.; FARIAS, A. A. Crescimento vegetativo do maracujazeiro-amarelo em diferentes tipos e dosagens de biofertilizante na forma líquida. Revista Verde, Mossoró, v. 3, n. 4, p. 116-122, 2008.

DELEITO, C. S. R.; CARMO, M. G. F.; ABBOUD, A. C. S.; FERNANDES, M. C. A. Sucessão microbiana durante o processo de fabricação do biofertilizante Agrobio. In: FERTBIO 2000 - REUNIÃO BRASILEIRA DE FERTILIDADEDOSOLOENUTRIÇÃODEPLANTAS, 25.; REUNIÃO BRASILEIRA SOBRE MICORRIZAS, 8.; SIMPOSIO BRASILEIRO DE MICROBIOLOGIA DO SOLO, 6.; REUNÃO BRASILEIRA DE BIOLOGIA DO SOLO, 3., 2000, Santa Maria. Anais... Santa Maria: Sociedade Brasileiro de Ciências do Solo e Sociedade Brasileiro de Microbiologia, 2000. CD-ROM.

DIAS, T. J.; PEREIRA, W. E.; SOUSA, G. G. Fertilidade de substratos para mudas de mangabeira, contendo fibra de coco e adubados com fósforo. Acta Scientiarum Agronomy, Maringá, v. 29, n. 5, p. 649-658, 2007.

EMPRESA BRASILEIRA DE PESQUISA AGROPECUÁRIA - EMBRAPA. Manual de análises químicas de solos, plantas e fertilizantes. 2. ed. Brasília: Embrapa Informação Tecnológica, 2009. 627 p.

FERREIRA, F. R. Recursos genéticos em passiflora. In: FALEIRO, F. G.; JUNQUEIRA, N. T. V.; BRAGA, M. F. (Org.). Maracujá - germoplasma e melhoramento genético. Planaltina: Embrapa Cerrados, 2005. p. 41-51.

FLARESSO, J. A.; GROSS, C. D.; ALMEIDA, E. X. Época e densidade de semeadura de aveia preta (Avena strigosa Schreb.) e azevém (Lolium multiflorum Lam.) no Alto Vale do Itajaí, Santa Catarina. Revista Brasileira de Zootecnia, Viçosa, v. 30, n. 6S, p. 1969-1974, 2001.

INSTITUTO AGRONÔMICO DO PARANÁ - IAPAR. Cartas climáticas do Paraná. Versão 1.0.2000. Londrina: IAPAR, 2000. CD-ROM.

INSTITUTO BRASILEIRO DE GEOGRAFIA E ESTATÍSTICA - IBGE. Produção agrícola municipal. 2011. Disponível em: <http://www.ibge.gov.br/home/ estatistica/economia/pam/2008/tab4.pdf>. Acesso em: 14 jan. 2011.

RBMC - Rede Brasileira de monitoramento contínuo, relatório de informação de estação, Estação Guarapuava - PRGU. 2009 Disponível em: <http:// www.geoftp.ibge.gov.br/RBMC/relatorio/ Descritivo_ PRGU.pdf $>$. Acesso em: 10 maio 2009. 
MINISTÉRIO DA AGRICULTURA, PECUÁRIA E ABASTECIMENTO - MAPA. Legislação brasileira. 2011. Disponível em: <http://www.prefiraorganicos. com.br/organicoshome.aspx $>$. Acesso em: 24 jan. 2011.

MALAVOLTA, E. Elementos de nutrição mineral de plantas. São Paulo: Ceres, 1980. 251 p.

MARSCHNER, H. Mineral nutrition of higher plants. Londres: Academic Press, 1995. 887 p.

MEINERZ, C. C.; MÜLlER, S. F.; SCHIMDT, M. A. H.; ECHER, M. M. Qualidade de mudas de couvechinesa em função de substratos e de adubações. In: CONGRESSO BRASILEIRO DE AGROECOLOGIA, 6.; CONGRESSO LATINOAMERICANO DE AGRoeCOlOGIA, 2., 2009, Curitiba. Resumos ... Porto Alegre: Revista Brasileira de Agroecologia, v. 4, n. 2, p. 3427-3431, 2009.

MELETTI, L. M. M. Maracujazeiro. In: MELETTI, L. M. M. (Org.). Propagação de frutíferas tropicais. Guaíba: Agropecuária, 2000. p. 189-204.

Renovação e poda do maracujá. 2011. Disponível em: <http://www.todafruta.com.br/portal/ icNoticiaAberta.asp?idNoticia $=18163>$. Acesso em: 25 jan. 2011.

MENDONÇA, V.; ABREU, N. A. A.; SOUZA, H. A.; FERREIRA, E. A.; RAMOS, J. D. Diferentes níveis de composto orgânico na formulação de substrato para a produção de mudas de mamoeiro 'formosa'. Caatinga, Mossoró, v. 20, n. 1, p. 49-53, 2007.

MENDONÇA, V.; MEDEIROS, L. F.; TOSTA, M. S.; MEDEIROS, P. V. Q.; OLIVEIRA, L. A. A. Sources alternative of organic matters for mix of substrates for the production of yellow-passion seedlings. Caatinga, Mossoró, v. 22, n. 2, p. 61-67, 2009.

NEGRETTI, R. R. D.; BINI, D. A.; AMARAL, U.; MARTINS, C. R. Avaliação da adubação orgânica em pimentão Capsicun annum cultivado em sistema orgânico de produção sob ambiente protegido. Revista da Faculdade de Zootecnia, Veterinária e Agronomia, Uruguaiana, v. 17, n. 1, p. 27-37, 2010.

PAUluS, G.; MÜLlER, A. M.; BARCELlOS, L. A. R. Agroecologia aplicada: práticas e métodos para uma agricultura de base ecológica. Porto Alegre: EMATER/ RS, 2001. 86 p.

PEIXOTO, J. R.; PÁDUA, T. de. Efeito da matéria orgânica do superfosfato simples e do cloreto de potássio na formação de mudas do maracujazeiro amarelo. Pesquisa Agropecuária Brasileira, Brasília, v. 24, n. 4, p. 417-422, 1989.

PIRES, A. A.; MONNERAT, P. H.; PINHO, L. G. R.;
ZAMPIROLLI, P. D.; ROSA, R. C. C.; MUNIZ, R. A. Efeito da adubação alternativa sobre os componentes de produção do maracujazeiro-amarelo. Acta Scientiarum Agronomy, Maringá, v. 31, n. 4, p. 655-660, 2009.

POSTIGLIONI, S. R. Comportamento da aveia, azevém e centeio na região dos Campos Gerais. Londrina: IAPAR, 1982. 18 p. (Boletim técnico, 14).

POTT, C. A.; MÜLlER, M. M. L.; BERTELLI, P. B. Adubação verde como alternativa agroecológica para recuperação da fertilidade do solo. Ambiência, Guarapuava, v. 3, n. 1, p. 51-63, 2007.

RAIJ, B. van; ANDRADE, J. C.; CANTARELLA, H.; QUAGGIO, J. A. Análise química para avaliação da fertilidade de solos tropicais. Campinas: IAC, 2001. 285 p.

RODRIGUES, A. C.; CAVALCANTE, L. F.; OLIVEIRA, A. P.; SOUZA, J. T.; MESQUITA, F. O. Produção e nutrição mineral do maracujazeiro-amarelo em solo com biofertilizante supermagro e potássio. Revista Brasileira de Engenharia Agrícola e Ambiental, Campina Grande, v. 13, n. 2, p. 117-124, 2009.

SERRANO, L. A. L.; SILVA, C. M. M.; OGLIARI, J.; CARVAlHO, A. J. C. C.; MARINHO, C. S.; DETMANN, E. Utilização de substrato composto por resíduos da agroindústria canavieira para produção de mudas de maracujazeiro-amarelo. Revista Brasileira de Fruticultura, Jaboticabal, v. 28, n. 3, p. 487-491, 2006.

SILVA, A. C. Avaliação da tolerância ao alumínio em plântulas de maracujazeiro e mamoeiro. 2009. Dissertação (Mestrado em Fitotecnia) - Instituto de Agronomia, Departamento de Fitotecnia. Universidade Federal Rural do Rio de Janeiro, Seropédica.

SILVA, E. A.; MARUYAMA, W. I.; MENDONÇA, V.; FRANCISCO, M. G. S.; BARDIVESSO, D. M.; TOSTA, M. S. Composição de substratos e tamanho de recipientes na produção e qualidade das mudas de maracujazeiro 'amarelo'. Ciência e Agrotecnologia, Lavras, v. 34, n. 3, p. 588-595, 2010.

SILVA, F. A. S. Assistat - assistência estatística. 2011. Disponível em: <http://www.assistat.com/indexp.html\# down>. Acesso em: 29 jun. 2011.

SILVEIRA, A. P. D.; SILVA, L. R.; AZEVEDO, I. C.; OLIVEIRA, G.; MELETTI, L. M. M. Desempenho de fungos micorrízicos arbusculares na produção de mudas de maracujazeiro-amarelo em diferentes substratos. Bragantia, Campinas, v. 62, n. 1, p. 89-99, 2003.

SOUZA, J. L. Pesquisas e tecnologias para a produção de hortaliças orgânicas. In: HORTIBIO 2001 - CONGRESSO BRASILEIRO DE HORTICULTURA ORGÂNICA, 
NATURAL, ECOLÓGICA E BIODINÂMICA, 1., 2001, Botucatu. Palestras... Botucatu: Agroecológica, 2001. p. 178-224.

SUSSEL, A. A. B. Importância da sanidade de mudas e de sua origem. Planaltina: Embrapa Cerrados, 2010. Disponível em: <http://www.cpac.embrapa.br/noticias/ artigosmidia/publicados/281/>. Acesso em: 20 dez. 2010.

TANAKA, M. T.; SENGIK, E.; SANTOS, H. S.; HABEL JÚNIOR, C.; SCAPIM, C. A.; SILVÉRIO,
L.; KVITSCHAL, M. V.; ARQUEZ, I. C. Efeito da aplicação foliar de biofertilizantes, bioestimulantes e micronutrientes na cultura do tomateiro (Lycopersicon esculetum Mill.). Acta Scientiarum Agronomy, Maringá, v. 25, n. 2, p. 315-321, 2003.

WILLER, H.; KLICHER, L. The world of organic agriculture - statistics and emerging trends 2009. Frick: FiBL, 2009. Disponível em: <www.organic-world.net>. Acesso em: 20 jan. 2011. 
